\title{
The peripheral zone of the prostate is more prone to tumor development than the transitional zone: Is the ETS family the key?
}

\author{
DAVID ADLER ${ }^{1}$, ANDREAS LINDSTROT ${ }^{1}$, JÖRG ELLINGER ${ }^{2}$, SEBASTIAN ROGENHOFER ${ }^{2}$, \\ REINHARD BUETTNER $^{1}$, SVEN PERNER $^{1}$ and NICOLAS WERNERT ${ }^{1}$ \\ ${ }^{1}$ Institute of Pathology, and ${ }^{2}$ Department of Urology, University Hospital Bonn, 53127 Bonn, Germany
}

Received July 9, 2011; Accepted October 7, 2011

DOI: $10.3892 / \mathrm{mmr} .2011 .647$

\begin{abstract}
Predisposition to develop prostate cancer (PCA) varies among the prostate zones, with the peripheral zone (PZ) more prone to tumor development than the transitional zone (TZ). In view of the fact that molecular differences between the zones may explain this difference, combined with the findings that translocations between TMPRSS2 and several ETS members are frequently observed in PCA, we hypothesized that the ETS family may be crucial to explaining this difference. Normal tissues from the PZ and the TZ of 20 PCA patients were laser microdissected to separate glands from stroma. Two oligo microarrays were performed in order to investigate the variation in ETS family gene expression between the glands and the stroma of the two zones. The ETS members, ELF-3, ELF-5, ERG, ETV-1, ETV-4, ETV-5, ETV-7 and FEV, were found to be differentially expressed. A striking observation was that ERG and ETV-1 were found to be up-regulated in the glands of the PZ compared to the TZ, particularly when considering that ERG and ETV-1 fusions account for 50-80\% and $20 \%$ of PCA occurrences, respectively. These results indicate that the glands and stroma of the two zones display distinct molecular differences and zonal-specific expression of ETS members. Furthermore, ETS members up-regulated in PCA are already overexpressed in the normal PZ, suggesting that these members play a role in the development and progression of PCA.
\end{abstract}

\section{Introduction}

Prostate cancer (PCA) is a clinically heterogeneous and often multifocal disease with a clinical outcome that is difficult to predict $(1,2)$. Therefore, advancements in the knowledge of the molecular basis of PCA should improve the prediction

Correspondence to: Professor Nicolas Wernert, Institute of Pathology, University Hospital Bonn, Sigmund Freud Str. 25, 53127 Bonn, Germany

E-mail: nicolas.wernert@ukb.uni-bonn.de

Key words: transcriptional regulation, stroma, glands, ETS, ERG of prognosis, as genetic aberrations drive the formation and aggressiveness of prostate carcinoma (3).

The prostate is supported by a stroma and can be divided into three anatomical zones: peripheral (the so-called prostate proper), transitional and central zones $(4,5)$. The predisposition to develop PCA is different among these zones, as most manifest PCAs (75\%) occur in the peripheral zone (PZ), at the dorsal and dorso-lateral side of the prostate, while only some $(20 \%)$ occur in the transitional zone (TZ) $(6,7)$.

The latter cancers are mostly so-called incidental carcinomas with a nonaggressive clinical course, whereas cancers from the $\mathrm{PZ}$ are more aggressive, tend to invade the periprostatic tissues and have higher biochemical recurrence rates $(6,8)$. The molecular basis underlying the differences in the susceptibility to PCA between the different zones of the prostate remains unknown. However, it has been suggested that some of the differences in susceptibility may have an embryological basis (9). As PCA rarely develops in the central zone compared with the PZ, it has been suggested that the central zone is derived from the Wolffian duct, the same duct from which the seminal vesicle is derived, and in contrast to the remainder of the prostate, which is derived from the urogenital sinus $(5,9,10)$.

In contrast to these varying predispositions of the $\mathrm{TZ}$ and the PZ to clinically manifest PCA, and to the fact that the two zones display no histomorphological differences (5), this suggests that there should be molecular differences explaining the various characteristics. A previous study reported that gene expression differences exist between the PZ and the TZ of the prostate and suggested that stromal-epithelial interactions may be responsible for the distinct zonal localization of PCA (11). It is already known that embryological prostatic development occurs through mesenchymal-epithelial interactions and is induced by androgens (12). The ability of the epithelial cells from the glands to respond to the stroma suggests that carcinoma development and progression may also be regulated by connective tissue and that the communication between the epithelium and stroma is multifactorial (12-14). Indeed, tumor-stroma communications consist of multiple dynamic interactions between neoplastic and stromal cells, and further evidence indicates that these communications also exist between metastatic PCA cell lines as well as their fibroblastic environment (15). 
Understanding prostatic carcinogenesis remains a major challenge and the discovery of the ETS family of oncoproteins in PCA within the last decade has been the greatest breakthrough towards this goal (4). In human PCA, translocations between the TMPRSS 2 gene, encoding an androgen-regulated, prostate-specific serine protease, with several genes of the ETS family including ETV-1, ETV-4, ETV-5 and, most commonly, ERG, are frequently reported (16-19). The TMPRSS2-ERG fusions appear to account for $50-80 \%$ of occurrences, while TMPRSS2-ETV-1 fusions account for up to $20 \%$ of occurrences $(4,20)$. The known fusion genes are thought to occur in up to $60-70 \%$ of clinically manifest PCA diseases (4). The consequence of such translocations is the increased expression of the rearranged ETS factors as a response to androgens in PCA (16). It has been suggested that such ETS rearrangements are sufficient for initiation of prostate neoplasia, while, most recently, other studies have suggested that the rearrangements may foster progression rather than initiation events in prostate tumorigenesis $(21,22)$.

In the present study, we hypothesized that the ETS family members may be the key to explaining the observation that the PZ of the prostate is more prone to tumor development than the TZ. Therefore, normal tissues from the PZ and the $\mathrm{TZ}$ of patients who had undergone radical prostatectomy for PCA were laser microdissected to separate the glands from the stroma. Two microarrays were then performed to investigate differences in ETS family gene expression between the glands and the stroma of the PZ and the TZ.

Our findings demonstrate that the glands and the stroma of the PZ and the TZ display distinct molecular differences, as well as zonal-specific expression of ETS members. Furthermore, the results also indicate that ETS members that are up-regulated in PCA are already overexpressed in the normal PZ, suggesting that these members may play a role in the development and progression of PCA. Taken together, these findings indicate that the ETS family members may be the key to explaining the observation that the $\mathrm{PZ}$ is more prone to tumor development than the TZ.

\section{Materials and methods}

The present study was approved by the Ethics Review Board of the Faculty of Medicine, University of Bonn/University Hospital, Germany, according to the principles expressed in the Declaration of Helsinki. Furthermore, written informed consent was obtained from all participants involved in the study.

All microarray data used in this study are MIAME compliant and the raw data have been deposited in a MIAME compliant database, GEO. The accession numbers for the manuscript are GSE29090.

Processing of human prostatectomy specimens. Radical prostatectomy specimens were obtained from PCA patients immediately following surgery. The patients were not exposed to any treatment prior to surgery. Fresh tissue samples $(0.5 \times 0.5 \times 0.3 \mathrm{~cm})$ were removed from the PZs (prostate proper) and the TZs of 20 PCA patients, and shock-frozen in liquid nitrogen with ice-cold isopentane. Frozen sections $(6 \mu \mathrm{m})$ were excised from the samples using a cryotome (Leica, Germany) and mounted on membrane-coated slides
(MembraneSlides, 1-mm PEN, Zeiss, Germany) for subsequent laser microdissection. One section was mounted on conventional slides and stained with hematoxylin and eosin (H\&E) for diagnostic evaluation by an experienced pathologist who confirmed the absence of prostate carcinoma in the two zones. Laser microdissection was performed as previously described (23-25). Frozen sections were dried for $2 \mathrm{~min}$ in the cryotome, washed for 2 min with $70 \%$ ethanol in DEPC-treated water and stained for $30 \mathrm{sec}$ in $1 \%$ cresyl violet diluted in $50 \%$ ethanol-DEPC-treated water. Slides were then washed briefly in $70 \%$ and $100 \%$ ethanol, dried for $10 \mathrm{~min}$ and stored at $-80^{\circ} \mathrm{C}$ until their use for laser microdissection of normal glands and the stroma between them.

Quality control. The quality of the RNA was measured from each patient prior to laser microdissection, using a lasercapture microscope (LCM). The section was washed from the slide with $600 \mu \mathrm{l}$ buffer RLT $+2 \mu \mathrm{M}$ DTT (provided in the Kit RNeasy Mini Kit, Qiagen, Germany) and vortexed for $30 \mathrm{sec}$. Then RNA extraction was performed as described according to the manufacturer's instructions. The recommended DNase digestion was created with a RNase-Free DNase Set (Qiagen). The quality of the RNA was measured with the Agilent Bioanalyzer 2100 (Agilent Technologies, CA, USA). Samples with a RIN factor $>6$ were used for LCM.

$L C M$. The cresyl violet-stained sections were cut with an Axio Observer.Z1 microscope (Zeiss) and installed Palm MicroBeam (Zeiss). The LCM was performed under a $\mathrm{x} 10$ objective. The glands and the stroma of the PZs and the TZs were separated and collected in $200 \mu \mathrm{l}$ AdhesiveCap tubes (Zeiss).

Microarray analysis of RNA isolated from lasermicrodissected healthy glands and stroma of the PZs and TZs of the prostate. RNA was isolated from laser microdissected healthy glands and stroma of the peripheral and TZs of the prostate from patients using the RNeasy Micro Kit (Qiagen) as described according to the manufacturer's instructions. The recommended DNase digestion was included with the RNase-Free DNase Set (Qiagen). The amount of the isolated RNA was measured with the Nanodrop photometer (Thermo Fisher Scientific, USA). Thereafter, an equal amount of RNA from healthy PZs and TZs of the glands of 10 patients was pooled, respectively, to a final concentration of $300 \mathrm{ng}$ of RNA. Furthermore, an equal amount of RNA was also taken from the stroma of healthy PZs and TZs of the patients and pooled, respectively, to a final concentration of $300 \mathrm{ng}$ of RNA. These pools were sent to MiltenyiBiotec (Bergisch Gladbach, Germany) for microarray analysis and bioinformatical interpretation. The RNA was labeled with $\mathrm{Cy} 3$ and hybridized on the whole human genome oligo microarray 4x44K (Agilent Technologies), according to the manufacturer's instructions. The microarray results were then validated by quantitative reverse transcriptase PCR (qRT-PCR) of a subset of genes.

\section{Results}

ETS family members are differentially expressed in normal glands of the $T Z$ compared to the $P Z$ of the prostate. Using 
whole human genome oligo microarray, we identified a set of 5/27 ETS family members to be differentially expressed $(\mathrm{p}<0.01)$ in the healthy glands of the PZ compared to TZ (Table I). We found ELF-5, ERG and ETV-1 to be up-regulated in normal glands of the PZ compared to the glands of TZ, whereas ETV-4 and ETV-5 were found to be down-regulated in the normal glands of the PZ compared to the TZ.

Differentially expressed ETS family members in the stroma of the TZ compared to the PZ of the prostate. A set of 5 ETS family members was found to be differentially expressed $(\mathrm{p}<0.01)$ in the stroma cells of the PZ compared to those of the TZ using whole human genome oligo microarray hybridization (Table II). We found FEV, ELF-3 and ERG to be up-regulated in the stroma of the PZ compared to those of the TZ. On the other hand, ETV-5 and ETV-7 were down-regulated in the stroma of the PZ compared to that of the $\mathrm{TZ}$.

\section{Discussion}

In this study, we examined the differences in gene expression of all ETS family members between the PZ and the TZ of the normal glands and stroma of the prostate. We report that 5/27 ETS family members were differentially expressed between the normal glands of the PZ compared to those of the TZ (Table I). Furthermore, 5/27 were found to be differentially expressed between the normal stroma of the PZ compared to that of the TZ (Table II). These results indicate that normal prostate glands and stroma of the PZ and the TZ display distinct molecular differences in regard to the expression of a unique set of ETS family members.

Previous studies have reported an overlap between genes that are overexpressed in prostate carcinoma and preferentially expressed in the normal PZ (11). In addition, tumor-associated genes were found to overlap with genes more highly expressed in the PZ compared to the TZ $(11,26)$. This overlap was suggested to be either a result of gene-expression signatures of the normal PZ that remains dominant in PCA, displaying its origin, or that the unique expression profile of the PZ shows a zonal-specific microenvironment that supports tumor growth (11). Based on these studies, it was proposed that genes that are up-regulated in prostate carcinoma are already overexpressed in the PZ, suggesting that these genes may play a role in the origin and progression of PCA (11). It was further suggested that the rarity of tumor development in the $\mathrm{TZ}$, might be due to the distinct expression profile in the TZ of specific genes and pathways that are involved in antitumor mechanisms (11).

Our results, which revealed that the ETS family members ERG and ETV-1, were up-regulated in the normal glands of the PZ compared to the TZ (Table I), combined with reports that ERG and ETV-1 fusions account for 50-80\% and for up to $20 \%$ of PCA occurrences, respectively $(4,20)$, support the proposed hypothesis of a predisposing cancer susceptibility signature in the PZ and a possible unique expression profile of the PZ microenvironment that supports tumor growth (11). This hypothesis can also be supported by our finding that ELF-5 is up-regulated in the glands of the PZ compared to the TZ (Table I), combined with previous studies reporting that increased expression of ELF-5 is associated with advanced
Table I. Differentially expressed ETS members in the normal glands of the PZ compared to the $\mathrm{TZ}$.

\begin{tabular}{lcc}
\hline ETS family member & PZ glands & TZ glands \\
\hline ELF-5 & +2.2 & 1 \\
ERG & +12.7 & 1 \\
ETV-1 & +2.2 & 1 \\
ETV-4 & -3.7 & 1 \\
ETV-5 & -2.3 & 1 \\
\hline
\end{tabular}

Values indicate the fold-change in gene expression with positive values indicating up-regulation and negative values indicate down-regulation. PZ, peripheral zone; TZ, transitional zone.

Table II. Differentially expressed ETS members in the normal stroma of the PZ compared to the TZ.

\begin{tabular}{lcc}
\hline ETS family member & PZ stroma & TZ stroma \\
\hline FEV & +2.4 & 1 \\
ELF-3 & +3.9 & 1 \\
ERG & +6.5 & 1 \\
ETV-5 & -2.1 & 1 \\
ETV-7 & -2.1 & 1 \\
\hline
\end{tabular}

Values indicate the fold-change in gene expression, with positive values indicating up-regulation and negative values indicating downregulation. $\mathrm{PZ}$, peripheral zone; $\mathrm{TZ}$, transitional zone.

stages of PCA (27). In addition, ETV-4, which is found to be down-regulated in the glands of the PZ compared to those of the TZ (Table I), is reported to have decreased expression or genomic loss that is associated with advanced stages of PCA (27). Notably, ERG is found to be up-regulated in the stroma of the PZ, as it is in the glands, along with its closely related ETS member FEV (28) and ELF-3 (Table II). In support of our hypothesis, ELF-3 is reported to have elevated expression in breast cancer (27).

Immunohistochemistry, and/or in situ hybridization studies, have reported on the expression of ETS family members in PCA (27). For instance, ERG, which we found to be up-regulated in the PZ compared to the $\mathrm{TZ}$, has been shown to be overexpressed in PCA tissues, whereas ETV-4, which we found to be down-regulated, was reported to show negative staining in PCA (27). We recently reported the expression pattern of all 27 ETS family members in androgen-sensitive VCaP and LNCaP, and androgen-insensitive PC3 and DU-145 PCA cell lines (29). We reported an expression specificity of various ETS family members in different cell lines (29). For instance, ERG, ETV-1 and ELF-5, which we found to be up-regulated in the PZ compared to the TZ, were found to be highly expressed in PC3 and DU-145 (for ERG) and in LNCaP (for ETV-1 and ELF-5) (29). Knockdown of ERG in PCA cell lines induced morphological changes and caused inhibition of cell growth in culture and mice (30). ETV-1 overexpression in benign prostate 
cells as well as in the mouse prostate conferred neoplastic phenotypes (21). Expression of ETV-4 leads to inhibition of cell growth and tumor development in cancer cells, suggesting a role as a tumor-suppressor gene (31).

Taken together, our findings indicate that the ETS family members, which are up-regulated in PCA, are already overexpressed in the normal PZ, suggesting that these ETS members may play a key role in the origin and progression of PCA and may explain the observation that the $\mathrm{PZ}$ is more prone to tumor development than the TZ.

Further studies must be performed to examine the transcriptional regulatory networks performed by various ETS family members in the normal prostate zones and in PCA, as well as the function of these genes. Ideally, it would be helpful to acquire prostate cell lines derived directly from the normal $\mathrm{PZ}$ and the TZ of the prostate, and to further examine the role of the ETS family members, as well as their potential targets and networks. These ETS candidates may then prove useful as diagnostic and prognostic markers, or even novel therapeutic targets, for PCA in the future.

\section{Acknowledgements}

This study was supported by a grant of the German Research Foundation [Deutsche Forschungsgemeinschaft (DFG)] (WE1104/11-1) and the German Cancer Aid (Deutsche Krebshilfe, 107827) to Nicolas Wernert, and by a grant of the German Research Foundation (DFG), Emmy Noether Program, (PE1179/2-1) and the Rudolf Becker Foundation to Sven Perner.

\section{References}

1. Arora R, Koch MO, Eble JN, Ulbright TM, Li L and Cheng L: Heterogeneity of Gleason grade in multifocal adenocarcinoma of the prostate. Cancer 100: 2362-2366, 2004.

2. Ruijter ET, van de Kaa CA, Schalken JA, Debruyne FM and Ruiter DJ: Histological grade heterogeneity in multifocal prostate cancer. Biological and clinical implications. J Pathol 180 295-299, 1996.

3. DeMarzo AM, Nelson WG, Isaacs WB and Epstein JI: Pathological and molecular aspects of prostate cancer. Lancet 361: 955-964, 2003.

4. Joshua AM, Evans A, Van der Kwast T, et al: Prostatic preneoplasia and beyond. Biochim Biophys Acta 1785: 156-181, 2008

5. McNeal JE: The zonal anatomy of the prostate. Prostate 2: 35-49, 1981.

6. McNeal JE, Redwine EA, Freiha FS and Stamey TA: Zonal distribution of prostatic adenocarcinoma. Correlation with histologic pattern and direction of spread. Am J Surg Pathol 12: 897-906, 1988 .

7. Guo CC, Zuo G, Cao D, Troncoso P and Czerniak BA: Prostate cancer of transition zone origin lacks TMPRSS2-ERG gene fusion. Mod Pathol 22: 866-871, 2009.

8. Colombo P, Patriarca C, Alfano RM, et al: Molecular disorders in transitional vs. peripheral zone prostate adenocarcinoma. Int J Cancer 94: 383-389, 2001.

9. Laczko I, Hudson DL, Freeman A, Feneley MR and Masters JR: Comparison of the zones of the human prostate with the seminal vesicle: morphology, immunohistochemistry, and cell kinetics. Prostate 62: 260-266, 2005.
10. Thiel R and Effert P: Primary adenocarcinoma of the seminal vesicles. J Urol 168: 1891-1896, 2002.

11. Van der Heul-Nieuwenhuijsen L, Hendriksen PJ, van der Kwast TH and Jenster G: Gene expression profiling of the human prostate zones. BJU Int 98: 886-897, 2006.

12. Cunha GR: Role of mesenchymal-epithelial interactions in normal and abnormal development of the mammary gland and prostate. Cancer 74: 1030-1044, 1994.

13. Cunha GR, Hayward SW and Wang YZ: Role of stroma in carcinogenesis of the prostate. Differentiation 70: 473-485, 2002.

14. Sung SY and Chung LW: Prostate tumor-stroma interaction: molecular mechanisms and opportunities for therapeutic targeting. Differentiation 70: 506-521, 2002.

15. Kaminski A, Hahne JC, El Haddouti M, Florin A, Wellmann A and Wernert N: Tumour-stroma interactions between metastatic prostate cancer cells and fibroblasts. Int J Mol Med 18: 941-950, 2006.

16. He J, Pan Y, Hu J, Albarracin C, Wu Y and Dai JL: Profile of Ets gene expression in human breast carcinoma. Cancer Biol Ther 6: 76-82, 2007.

17. Tomlins SA, Rhodes DR, Perner S, et al: Recurrent fusion of TMPRSS2 and ETS transcription factor genes in prostate cancer. Science 310: 644-648, 2005.

18. Furusato B, Tan SH, Young D, et al: ERG oncoprotein expression in prostate cancer: clonal progression of ERG-positive tumor cells and potential for ERG-based stratification. Prostate Cancer Prostatic Dis 13: 228-237, 2010.

19. Kumar-Sinha C, Tomlins SA and Chinnaiyan AM: Recurrent gene fusions in prostate cancer. Nat Rev 8: 497-511, 2008.

20. Cai C, Hsieh CL, Omwancha J, et al: ETV1 is a novel androgen receptor-regulated gene that mediates prostate cancer cell invasion. Mol Endocrinol 21: 1835-1846, 2007.

21. Tomlins SA, Laxman B, Dhanasekaran SM, et al: Distinct classes of chromosomal rearrangements create oncogenic ETS gene fusions in prostate cancer. Nature 448: 595-599, 2007.

22. Carver BS, Tran J, Chen Z, et al: ETS rearrangements and prostate cancer initiation. Nature 457: E1-3, 2009.

23. Wellmann A, Wollscheid V, Lu H, et al: Analysis of microdissected prostate tissue with ProteinChip arrays: A way to new insights into carcinogenesis and to diagnostic tools. Int J Mol Med 9: 341-347, 2002.

24. Okuducu AF, Janzen V, Hahne JC, Ko Y and Wernert N: Influence of histochemical stains on quantitative gene expression analysis after laser-assisted microdissection. Int J Mol Med 11: 449-453, 2003.

25. Okuducu AF, Hahne JC, Von Deimling A and Wernert N: Laser-assisted microdissection, techniques and applications in pathology (Review). Int J Mol Med 15: 763-769, 2005.

26. Stuart RO, Wachsman W, Berry CC, et al: In silico dissection of cell-type-associated patterns of gene expression in prostate cancer. Proc Natl Acad Sci USA 101: 615-620, 2004.

27. Watson DK, Turner DP, Scheiber MN, Findlay VJ and Watson PM: ETS transcription factor expression and conversion during prostate and breast cancer progression. Open Cancer J 3: 24-39, 2010.

28. Peter M, Couturier J, Pacquement $\mathrm{H}$, et al: A new member of the ETS family fused to EWS in Ewing tumors. Oncogene 14: 1159-1164, 1997.

29. Adler D, Lindstrot A, Langer B, Buettnerand R and Wernert N: Differential expression of ETS family members in prostate cancer tissues and androgen-sensitive and -insensitive prostate cancer cell lines. Int J Mol Med 28: 89-93, 2011.

30. Sun C, Dobi A, Mohamed A, et al: TMPRSS2-ERG fusion, a common genomic alteration in prostate cancer activates C-MYC and abrogates prostate epithelial differentiation. Oncogene 27: 5348-5353, 2008.

31. Xing X, Wang SC, Xia W, et al: The ets protein PEA3 suppresses HER-2/neu overexpression and inhibits tumorigenesis. Nat Med 6: 189-195, 2000. 